\title{
GLDPC Codes with Reed-Muller Component Codes Suitable for Optical Communications
}

\author{
Ivan B. Djordjevic, Member, IEEE, Lei Xu, Ting Wang, and Milorad Cvijetic
}

\begin{abstract}
In this paper, we consider the GLDPC codes with Reed-Muller (RM) and Bose-Chaudhuri-Hocquenghem (BCH) codes as component codes. GLDPC codes with RM codes as component codes is an attractive option for high-speed optical transmission because they provide excellent coding gains, while the RM codes can be decoded using low-complexity maximum a posteriori probability (MAP) decoding based on fast WalshHadamard transform. Several classes of GLDPC codes (with component RM or BCH codes) outperforming the turbo product codes in terms of decoding complexity and coding gain are presented. We also identify several turbo product codes suitable for use in optical communications.
\end{abstract}

Index Terms-Optical communications, Reed-Muller (RM) codes, BCH codes, generalized low-density parity-check codes, Walsh-Hadamard transform, turbo-product codes.

\section{INTRODUCTION}

$\mathbf{T}$ HE state-of-the-art fiber-optics communication systems standardized by the ITU employ different concatenated Bose-Chaudhuri-Hocquenghem $(\mathrm{BCH}) /$ Reed-Solomon (RS) codes [1]-[3]. Recently, iteratively decodable codes, turbo [2] and low-density parity-check (LDPC) codes [3], have generated significant research attention. It has been shown in [3] that turbo product codes (TPCs) can be matched and outperformed by LDPC codes in terms of coding gain and decoding complexity. Generalized low-density parity-check (GLDPC) coding [3]-[6] can further improve the overall characteristics of LDPC codes by decreasing the complexity of decoder, which is of high importance for optical communications. The main idea behind the GLDPC codes is to replace the paritycheck equations in a parity-check matrix of global LDPC code by a linear block code (see [3]-[6] for more details). The decoding is based on a combination of simple and fast soft-input-soft-output (SISO) linear block decoders operating in parallel. The bit reliabilities obtained by SISO decoders are passed to the message-passing decoder operating on a bipartite graph of a global LDPC code. The SISO decoders are commonly designed as maximum a posteriori probability (MAP) decoders, such as Bahl-Cocke-Jelinek-Raviv (BCJR) decoder [7], and provide accurate estimates of bit reliabilities for a global LDPC decoder after small number of iterations. Due to high-complexity of the BCJR decoder, the GLDPC coding is limited to simple linear block component codes such as Hamming codes. The applications with Hamming codes as component codes are considered in [3],[5],[6].

Manuscript received April 14, 2008. The associate editor coordinating the review of this letter and approving it for publication was C. Comaniciu. This paper was supported in part by the NSF under Grant IHCS-0725405.

I. B. Djordjevic is with the Department of Electrical and Computer Engineering, University of Arizona, Tucson, AZ 85721 (e-mail: ivan@ece.arizona.edu).

L. Xu and T. Wang are with NEC Laboratories America, Princeton, NJ 08540 .

M. Cvijetic is with NEC Corporation of America, Herndon, VA 20171.

Digital Object Identifier 10.1109/LCOMM.2008.080590.
In this paper, which is a different version of the previous work on GLDPC codes with Hamming component codes [3], $\mathrm{BCH}$ and RM codes are employed as component codes. Notice that GLDPC codes with BCH component codes are considered in [8], but for hard decision decoding only. GLDPC coding with RM component codes is an attractive option for highspeed optical transmission because it can utilize an efficient low-complexity MAP decoding algorithm based on the modified Walsh-Hadamard transform proposed in [9] instead of high-complexity BCJR decoder. Several classes of GLDPC codes suitable for high-speed implementation are introduced. $\operatorname{RM}(4,6)$ based GLDPC $(4096,3201,0.78,16)$ code (the parameters of code represent codeword length, information word length, code rate, and lower-bound on minimum distance, respectively), and $\mathrm{BCH}(63,57)$ based $\operatorname{GLDPC}(6048,4896,0.81$, 9) code of code rate 0.81 outperform TPC (employing Chase II algorithm operating on 3 least reliable bit positions) by $0.93 \mathrm{~dB}$ on an additive white Gaussian noise (AWGN) channel model at BER of $10^{-9}$. BER performances are also assessed using an advanced fiber-optics channel model. We also identify several classes of turbo-product codes with $\mathrm{RM} / \mathrm{BCH}$ component codes suitable for use in optical communications.

\section{GLDPC CODES/TPCS WITH RM/BCH COMPONENT CODES}

To construct a GLDPC code, one can replace each single parity-check equation of a global LDPC code by the paritycheck matrix of a simple linear block code, known as the constituent (local) code, and this construction is proposed by Lentmaier and Zigangirov [6], and we will refer to this construction as LZ-GLDPC code construction. In another construction proposed by Boutros et al. in [5], referred here as B-GLDPC code construction, the parity-check matrix, $H$, is a sparse matrix partitioned into $W$ sub-matrices $H_{1}, \ldots, H_{W}$. $H_{1}$ is a block-diagonal matrix generated from an identity matrix by replacing the ones by a parity-check matrix of a local code of codeword-length $n$ and dimension $k$. Each submatrix $H_{j}$ is derived from $H_{1}$ by random column permutations. For more details on LZ-GLDPC and B-GLDPC like codes, and their generalization-fractal GLDPC codes (a local code is another GLDPC code) an interested reader is referred to [3]. The code rate of a GLDPC code is lower bounded by $R=K / N \geq 1-W(1-k / n)$, where $K$ and $N$ denote the dimension and the codeword-length of a GLDPC code, $W$ is the column weight of a global LDPC code, and $k / n$ is the code rate of a local code ( $k$ and $n$ denote the dimension and the codeword-length of a local code). The GLDPC codes with component Hamming codes are considered in [3], here we are concerned with GDLPC codes with component codes based on $\mathrm{RM}$ or $\mathrm{BCH}$ codes.

A Reed-Muller code $\operatorname{RM}(r, m)$ of order $r$ and codeword length $n=2^{m}$ [1] is the set of all binary vectors associated 
with coefficients of Boolean polynomials of degree at most $r$ in $m$ variables. The minimum distance of $\operatorname{RM}(r, m)$ code is $2^{m-r}$, and the dimension is determined as [1]

$$
k=1+\left(\begin{array}{c}
m \\
1
\end{array}\right)+\ldots+\left(\begin{array}{c}
m \\
r
\end{array}\right)
$$

An interesting property of RM codes is that they can be defined recursively [1]: $R M(r, m)=\{(a \mid a+b): a \in$ $R M(r, m-1), b \in R M(r-1, m-1)\}$, where $(x \mid y)$ denotes the concatenation operation. The generator matrix of $\operatorname{RM}(r, m)$ code, denoted as $G(r, m)$, can be therefore defined recursively by

$$
G(r, m)=\left[\begin{array}{cc}
G(r, m-1) & G(r, m-1) \\
0 & G(r-1, m-1)
\end{array}\right]
$$

and can be observed as two-level generalized concatenated code [1]. $\operatorname{RM}(0, m)$ is a repetition code, $\operatorname{RM}(m-1, m)$ is a parity-check code, and $\operatorname{RM}(m, m)$ corresponds to $2^{m}$-tuples of a vector space. The generator matrix of $\operatorname{RM}(m, m)$ can be represented by

$$
G(m, m)=\left[\begin{array}{ccccc} 
& G(m-1, m) & & & \\
0 & 0 & \ldots & 0 & 1
\end{array}\right]
$$

Another interesting property of RM codes is that the dual of $\operatorname{RM}(r, m)$ code is another $\operatorname{RM}(m-r-1, m)$ code. Therefore, the generator matrix of $\operatorname{RM}(m-r-1, m)$ code can be used as the parity check matrix of $\operatorname{RM}(r, m)$ code. If the recursion (2) is applied successively several times the $\operatorname{RM}(r, m)$ can be decomposed into several parity-check codes $\operatorname{RM}\left(m^{\prime}-1, m^{\prime}\right)$, repetition codes $\operatorname{RM}\left(0, m^{\prime}\right)$, and the firstorder $\operatorname{RM}\left(1, m^{\prime}\right)$ codes. The MAP decoding of parity-check or repetition codes is trivial, while the first order $\mathrm{RM}\left(1, m^{\prime}\right)$ codes can be decoded using an efficient MAP decoding algorithm proposed in [9], based on fast Hadamard-Walsh transform (FHWT). The overall complexity of that algorithm is in order $n^{\prime} \log _{2} n^{\prime}$ (where $n^{\prime}=2^{m^{\prime}}$ denotes the codeword length of the first order $\operatorname{RM}\left(1, m^{\prime}\right)$ component code), which is significantly lower than complexity of the BCJR algorithm that requires about $n^{n-k+1}$ operations [7]. Therefore, the complexity of GLDPC codes with RM component codes is of order $N \log _{2} n$. Since the complexity of sum-product algorithm is of order $\left(N_{L D P C}-K_{L D P C}\right) w_{r}$, with $w_{r}$ being the row weight of LDPC code parity check matrix, by proper selection of global LDPC code length $N$ and local RM code length $n$, the complexity of GLDPC codes is about $\left(N_{L D P C}-\right.$ $\left.K_{L D P C}\right) w_{r} /\left[(N / n) \sum\left(n^{\prime} \log _{2} n^{\prime}\right)\right]\left(n^{\prime}<n\right)$ times lower. For example, RM $(4,6)$ code can be decomposed using Eq. (2) on $\operatorname{RM}(1,2), \operatorname{RM}(1,3), \operatorname{RM}(2,2), \operatorname{RM}(3,3)$, and $\operatorname{RM}(4,4)$ component codes. Decoding of $\operatorname{RM}\left(m^{\prime}, m^{\prime}\right)\left(m^{\prime}=1,2,3,4\right)$ is trivial while the complexity of $\operatorname{RM}(4,6)$ is dominated by complexity of $\operatorname{RM}(1,3)$ decoding block and three $\operatorname{RM}(1,2)$ blocks, which is of order $\sum\left(n^{\prime} \log _{2} n^{\prime}\right)=8 \log _{2} 8+3 \cdot 4 \log _{2} 4=48$. B-GLDPC code with $W=2$ and length $N=4096$ based on $\mathrm{RM}(4,6)$ code has therefore complexity 11 times lower than girth-8 column-weight-4 LDPC code of length 8547 (and row weight 21) considered in Section III. Notice also that GLDPC decoder for 4096 code contains 4096/64=64 decoder blocks [composed of $\operatorname{RM}(1,2), \operatorname{RM}(2,3)$ and $\operatorname{RM}\left(m^{\prime}, m^{\prime}\right)\left(m^{\prime}=\right.$ $1, \ldots, 4)$ decoders], operating in parallel, and this structure is suitable for FPGA or VLSI implementation. The minimum distance of GLDPC codes is lower bounded by Tanner's inequality [4]. From that inequality we can conclude that large girth leads to an exponential increase in the minimum distance, and large minimum distance of the local code $d$ leads to an increase of the base. In order to keep the code rate high, in all simulations presented in Section III, we have selected columnweight of a global code to be $W=2$. In that case, if the girth of global code is $g=8$, the minimum distance of GLDPC code is simply $D \geq d^{2}$.

For the description of $\mathrm{BCH}$ codes an interested reader is referred to [1]. Notice that $\mathrm{BCH}$ codes can be decoded using an efficient MAP algorithm proposed in [9] with complexity $n /\left[(n-k) \log _{2} n\right]$ times lower than that of BCJR algorithm (see [7],[9] for more details about BCJR algorithm).

The TPCs based on BCH component codes are intensively studied for fiber-optics communications [2]. Notice that a TPC can be considered as a special case of B-GLDPC code composed of two sub-matrices $H_{1}$ and $H_{2}$ corresponding to parity-check matrices of outer and inner component codes, respectively; with permutation operation implemented using a block-interleaver approach. The TPCs can be decoded by sequentially decoding the columns and rows using the BCJR algorithm. However, due to high-complexity of BCJR algorithm, the sub-optimal low-complexity Chase II algorithm is commonly employed in practical applications [2]. However, if inner code is based on RM codes, it can be decoded by employing Ashikhmin's algorithm [9], which provides accurate estimates of initial log-likelihood ratios. For example, the product $\mathrm{BCH}(512,484) \times \mathrm{RM}(5,6)$ (of rate $R=0.93$ ) can be decoded using the MAP algorithm as inner $\operatorname{RM}(5,6)$ decoder $(\operatorname{RM}(5,6)$ code is in fact a simple parity check equation), and Chase II algorithm as outer $\operatorname{BCH}(512,484)$ decoder. Another interesting example is $\mathrm{RM}(6,8) \times \mathrm{RM}(5,7)(R=0.905)$ product code. The inner RM(5,7) decoder can be implemented using Ashikhmin's MAP decoding algorithm, while the outer $\mathrm{RM}(6,8)$ decoder can be implemented using Chase II algorithm. Both examples are suitable for terrestrial long-haul optical transmission systems. Turbo product example suitable for submarine systems is $\mathrm{BCH}(128,113) \times \mathrm{BCH}(256,239) \mathrm{TPC}$. It has been shown in [10] that the complexity of TPC with extended Hamming component code is comparable to $R=0.8$ LDPC code, if fast Chase II algorithm [10] is employed. However, the complexity of standard Chase II algorithm is about 5 times larger than that of fast Chase II algorithm for $p=3$ [10], and consequently the complexity of TPCs with $\mathrm{BCH}$ component codes is higher than complexity of LDPC decoder.

The GLDPC codes proposed in this paper can be put in systematic form, so that the efficient encoding algorithm due to Zhang and Parhi [12] can be employed. This algorithm can efficiently be performed in general purpose digital signal processors, as shown in [12].

\section{Performance Analysis and Conclusion}

The results of simulation for the AWGN channel model are shown in Fig. 1, and are obtained by maintaining the doubleprecision. GLDPC codes based on $\operatorname{BCH}(63,57)$ and $\mathrm{RM}(4,6)$ component codes for $W=2$ perform comparably. The $\mathrm{RM}(4,6)$-based GLDPC code outperforms the $\mathrm{BCH}(128,113) \mathrm{x}$ $\operatorname{BCH}(256,239)$ TPC based with Chase II decoding algorithm on $p=3$ least reliable bit positions by $0.93 \mathrm{~dB}$ at BER 

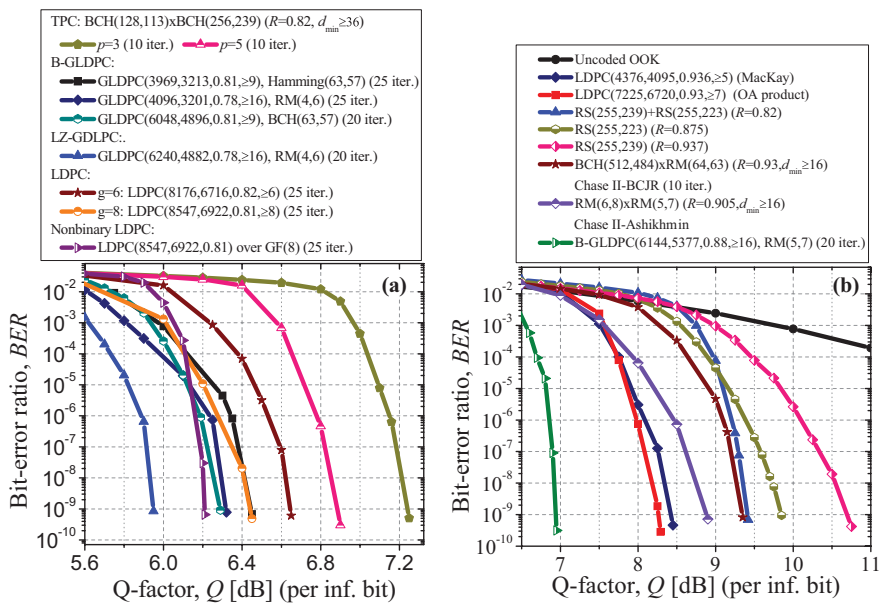

Fig. 1. BER performance on an AWGN channel: (a) GLDPC codes against TPCs and LDPC codes, (b) high-rate codes.

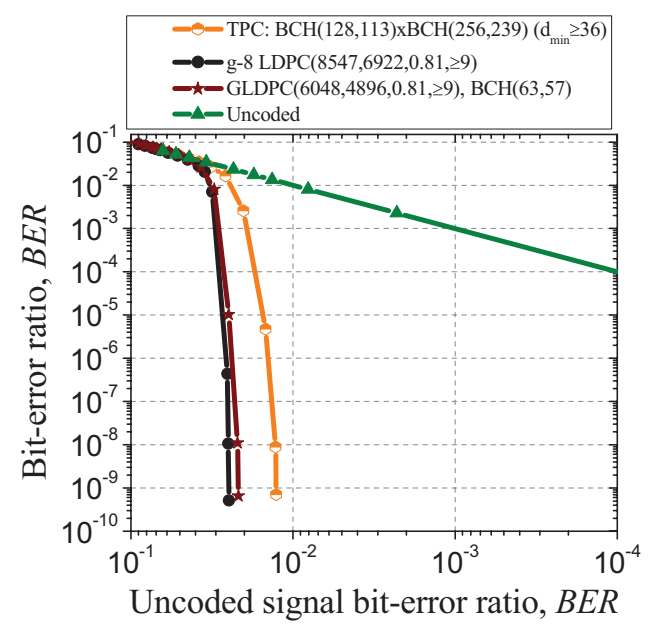

Fig. 2. GLDPC codes against TPC and LDPC codes at $40 \mathrm{~Gb} / \mathrm{s}$.

of $10^{-9}$ (see Fig. 1(a)). Notice that similar TPC was implemented in LSI technology (see [2] for more details). The TPC codeword is significantly longer, and the decoding complexity of GLDPC code based on $\operatorname{RM}(4,6)$ is at least 10 lower. During decoding TPC decoder employs 239 Chase II blocks operating in parallel, while GLDPC code on $\operatorname{RM}(4,6)$ code requires only 64 low-complexity MAP decoders as explained in Section II. In simulations presented here we have employed an efficient realization of Chase II algorithm proposed in [10]. In Fig. 1(b) BER performance of several classes of iteratively decodable codes (TPCs, LDPC and GLDPC codes) of high code rate are compared against conventional RS, and concatenated RS codes. B-GLDPC code of rate 0.88 outperforms concatenated RS code of rate 0.82 by $2.47 \mathrm{~dB}$ (also at $\left.B E R=10^{-9}\right) . \mathrm{RM}(6,8) \times \mathrm{RM}(5,7) \mathrm{TPC}$ of rate 0.905 outperforms concatenated RS code $(R=0.82)$ by $0.53 \mathrm{~dB}$ at $B E R$ of $10^{-9}$. LDPC code of rate 0.93 , designed using the concept of product of orthogonal arrays (OAs), outperforms the same RS concatenated code by $1.15 \mathrm{~dB}$ at $B E R$ of $10^{-9}$. $R=0.93$ LDPC code outperforms RS code of rate 0.937 by $2.44 \mathrm{~dB}$ at BER of $10^{-9}$.

The results of simulations for the single channel transmission at $40 \mathrm{~Gb} / \mathrm{s}$, and dispersion map similar to the from [3], are shown in Fig. 2. The span length is set to $L=120 \mathrm{~km}$, and each span consists of $2 L / 3 \mathrm{~km}$ of $\mathrm{D}+$ fiber followed by $L / 3 \mathrm{~km}$ of $\mathrm{D}$ - fiber. Pre-compensation of $-320 \mathrm{ps} / \mathrm{nm}$ and corresponding post-compensation are also applied. RZ modulation format of a duty cycle of 0.33 is observed, and the launched power is set to $0 \mathrm{dBm}$. The GLDPC code of rate 0.81 and codeword length 6048 with $\mathrm{BCH}(63,57)$ code as component code, performs slightly worse than girth-8 LDPC code of the same rate and codeword length 8547. However, decoding complexity of GLDPC code is lower. Both GLDPC and LDPC codes, although of lower complexity, outperform $\mathrm{BCH}(128,113) \times \mathrm{BCH}(256,239)$ turbo product code of length 32768 and code rate 0.824 . Notice that RM-based GLDPC codes require larger number of iterations than corresponding TPCs. On the other hand, the decoding algorithm of RM codes is based on FHWT, which for sequence of length $n$ requires $n \log _{2} n$ multiplications and additions, comparable to that of the fast Fourier transform (FFT).

Notice that parallel concatenated convolutional codes (PC$\mathrm{CCs}$ ), often used in wireless communications, have not been considered for use in optical communications because the PCCCs exhibit error floor phenomena around $10^{-6}$ (see [11]), while the targeted BERs in optical communications are well below $10^{-9}$ (often $10^{-15}$ ).

In conclusion, GLDPC codes with BCH and RM component codes are considered as possible options for high-speed optical transmission. It is demonstrated by simulation that the RM or BCH based GLDPC codes are able to outperform their turbo product counterparts in terms of coding gain with lower complexity in decoding algorithm. On the other hand, they require larger number of iterations. GLDPC codes perform comparably to girth-8 LDPC codes, but have lower complexity. Several classes of GLDPC codes and turbo-product codes suitable for use in both free-space optical communications and fiber-optics communications are presented.

\section{REFERENCES}

[1] R. H. Morelos-Zaragoza, The Art of Error Correcting Coding. Boston, MA: John Wiley and Sons, 2002.

[2] T. Mizuochi et al., "Forward error correction based on block turbo code with 3-bit soft decision for $10 \mathrm{~Gb} / \mathrm{s}$ optical communication systems," IEEE J. Select. Top. Quantum Electron., vol. 10, pp. 376-386, 2004.

[3] I. B. Djordjevic, O. Milenkovic, and B. Vasic, "Generalized low-density parity-check codes for optical communication systems," IEEE/OSA J. Lightwave Technol., vol. 23, pp. 1939-1946, May 2005.

[4] R. M. Tanner, "A recursive approach to low complexity codes," IEEE Trans. Inform. Theory, vol. IT-27, pp. 533-547, Sept. 1981.

[5] J. Boutros, O. Pothier, and G. Zemor, "Generalized low density (Tanner) codes," in Proc. IEEE Int. Conf. Comm. (ICC'99), pp. 441-445, 1999.

[6] M. Lentmaier and K. Sh. Zigangirov, "On generalized low-density paritycheck codes based on Hamming component codes," IEEE Commun. Lett., vol. 3, pp. 248-250, Aug. 1999.

[7] L. R. Bahl, J. Cocke, F. Jelinek, and J. Raviv, "Optimal decoding of linear codes for minimizing symbol error rate," IEEE Trans. Inform. Theory, vol. IT-20, no. 2, pp. 284-287, Mar. 1974.

[8] N. Miladinovic, and M. Fossorier, "Generalized LDPC codes with ReedSolomon and BCH codes as component codes for binary channels," in Proc. GLOBECOM '05, vol. 3, pp. 1239-1244, 2005.

[9] A. Ashikhmin and S. Lytsin, "Simple MAP decoding of first-order ReedMuller and Hamming codes," IEEE Trans. Inform. Theory, vol. 50, pp. 1812-1818, Aug. 2004.

[10] S. A. Hirst et al., "Fast Chase algorithm with an application in turbo decoding," IEEE Trans. Commun., vol. 49, pp. 1693-1699, 2001.

[11] W. E. Ryan, "Concatenated codes and iterative decoding," in Wiley Encyclopedia of Telecommunications (J. G. Proakis, ed.) New York: Wiley and Sons, 2003.

[12] T. Zhang and K. K. Parhi, "A class of efficient-encoding generalized low-density parity-check codes," in Proc. ICASSP '01, vol. 4, pp. 24772480, 2001. 